\title{
A Low-Complexity C-RS-Aided Channel Estimation Scheme for LTE Downlink System
}

\author{
Mohammed Zourob, and Dr. Raveendra Rao \\ Faculty of Engineering, Department of Electrical and Computer Engineering, \\ The University of Western Ontario, London, ON, Canada, N6A 3K7 \\ \{mzourob, rrao\}@uwo.ca
}

\begin{abstract}
GPP Long Term Evolution (LTE) downlink (DL) systems employ channel estimation algorithms in order to help with coherent detection. In this paper, we are proposing a new scheme for Cell-Specific Reference Signals (CRS)-aided channel estimation, which is 2 1-D Wiener filtering with linear interpolation as a less computationally-complex option compared to 2-D Wiener filtering and interpolation. Moreover, we present mathematical equations that describe C-RS symbols extraction from the Resource Grids (RG) for antenna ports $0,1,2$ and 3 . Simulations shows that 2 1-D Wiener filtering with linear interpolation requires $\sim 41 \%$ of the number of computations needed by $2-\mathrm{D}$ Wiener filtering with linear interpolation. Moreover, simulations indicate that the best noise reduction method is a combination of both averaging and Wiener filtering with linear interpolation, where the lower bound is a function of both SNR and the channel statistics.
\end{abstract}

Keywords: LTE, LTE Advanced, channel estimation, C-RS-aided channel estimation, Wiener interpolation, OFDM.

\section{Introduction}

Most telecommunication operators all over the world recognize the third generation partnership project (3GPP) long term evolution (LTE) as the internationally powerful mobile communication system [1]. LTE aims for significantly increased peak data rates, cell-edge user throughput efficiency, and higher average spectrum efficiency [2]. In order to meet the demand, some advanced technologies, such as multiple-inputmultiple-output (MIMO) and Orthogonal Frequency Division Multiplexing (OFDM), have been adopted by the LTE physical layer.

OFDM has been used in the physical layer of the LTE DL systems due to its high bandwidth efficiency and its ability to combat multi-path fading channels. For many coherent detection systems, a dynamic channel estimation algorithm is necessary for the design of OFDM receivers [3], [4]. The receiver could estimate the whole channel response of each OFDM symbol by processing the received symbols at predefined positions with known transmitted values. Different reference signals are transmitted in LTE system for different purposes. C-RS are transmitted on DL antenna ports $0,1,2$ and 3 and they are used for cell search and initial acquisition, DL channel quality measurements and for coherent demodulation/detection. These antenna ports do not correspond to physical antennas, but rather are logical entities distinguished by their 
reference signal sequences. Multiple antenna port signals can be transmitted on a single transmit antenna. Such kind of C-RS-aided channel estimation has been proven as a feasible method for OFDM systems. C-RS-aided channel estimation algorithms can be based on Least Square (LS) or Linear Minimum Mean Square Error (LMMSE) [4], where LS is used to estimate pilot symbols and LMMSE is used to enhance the estimation by reducing the noise effect using the channel's statistical information. LS is relatively simple, which dose not need relevant channel information and is easily affected by noise. As for LMMSE, channel response is estimated with the help of channel statistic information and relevance between subcarriers. However, it is very computationally complex compared to LS.

Related previous works in the literature uses the LMMSE for both noise reduction and interpolation. However, the associated complexity of using LMMSE for both estimation and interpolation is still high. Thus, we have previously proposed a new implementation scheme that uses 2-D Wiener filtering, which is based on the MMSE theory, to reduce the noise effect on the LS estimated pilots only, and then linear interpolates the data symbols. That was proposed as a sub-optimum scheme instead of using 2-D Wiener filtering with Wiener interpolation. In this paper, we propose using 2 1-D Wiener filtering with linear interpolation to further reduce the computational complexity of the system. Performance evaluation and complexity analysis in estimating LTE C-RS at ports 0, 1, 2, and 3 are reported and compared to 2-D Wiener filtering and Equal Weight Averaging (EWA) with linear interpolation.

The rest of the paper is organized as follows: LTE DL system model is introduced in Section 2, and some channel estimation algorithms are presented in Section 3. Simulation results and discussions are presented in Section 4, followed by Section 5 which concludes this paper.

\section{LTE Downlink System Model}

Fig. 1 shows the OFDM system block diagram and the C-RS pilots insertion. $x(k)$ is the input data at the transmitter and $y(k)$ is the output data at the receiver. At the transmitter, $x(k)$ is mapped using one of the many mappers available (BPSK, QPSK, QAM...etc) and transformed into an $N$-point sequence $x(n)$, where $N$ is the length of Inverse Fast Fourier Transform (IFFT). A Cyclic Prefix (CP) is added before transmitting in order to mitigate Inter Symbol Interference (ISI). At the receiver side, the channel estimation is done after performing Fast Fourier Transform (FFT), and it consists of three stages: equalization, noise reduction and interpolation.

3GPP LTE supports two radio frame structures. One is applicable to both full duplex and half duplex FDD system, which is shown in Fig .2. The other is applicable to TDD. In Fig. 2, data is transmitted in packets, called radio frames, between one Base Station (BS) and one user equipment (UE). Each radio frame consists of 10 sub-frames and spanning $10 \mathrm{~ms}$ in time. Each sub-frame occupies $1 \mathrm{~ms}$ in time and consists of two time slots. The OFDM frequency and time plane is called a resource grid, and each plane spanning 12 subcarriers and 14 OFDM symbols is called a Resource Block (RB). In each $\mathrm{RB}$, there are $12 \times 14=168$ Resource Elements $(\mathrm{RE})$. 


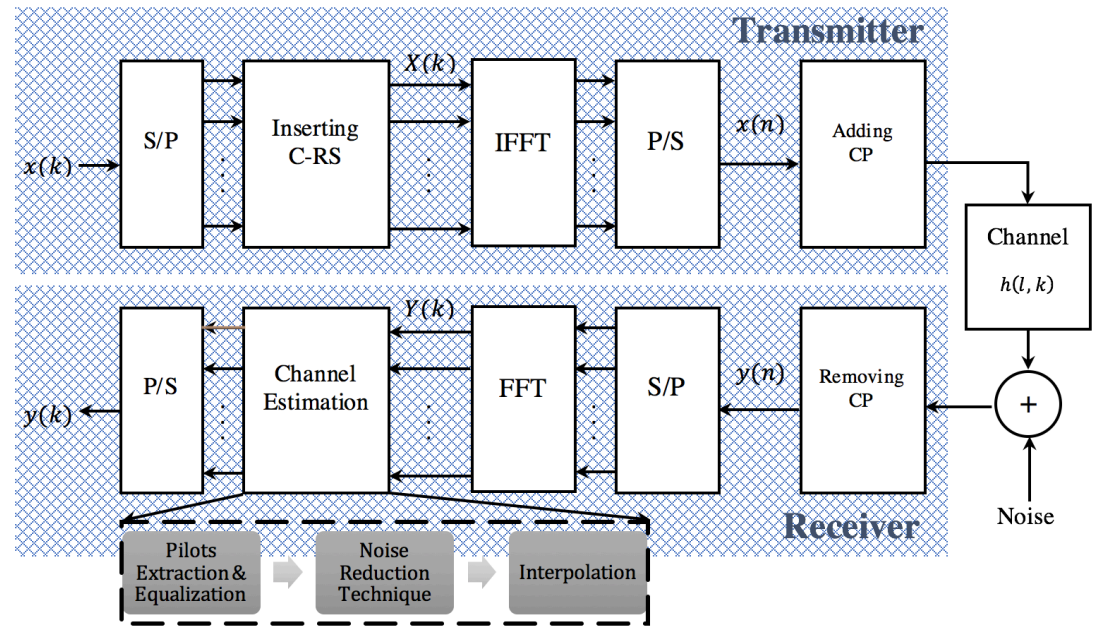

Fig. 1. Block diagram of an OFDM system

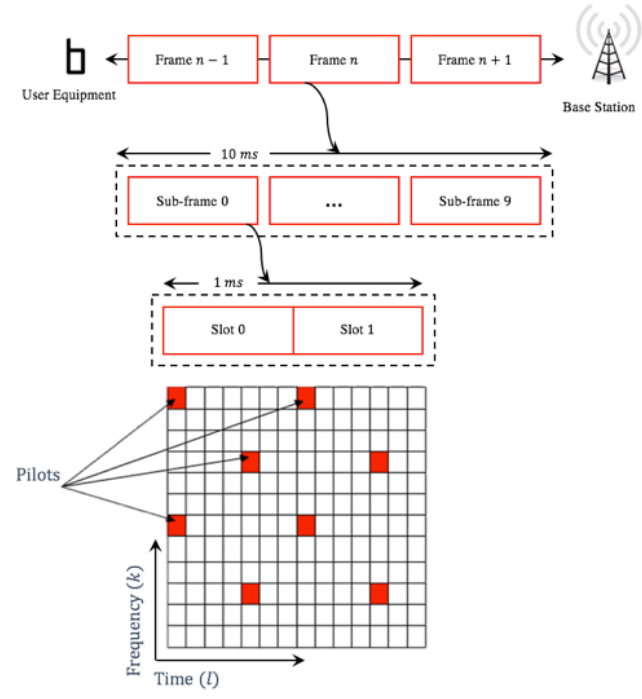

Fig. 2. Frame structure in LTE FDD

In LTE DL, C-RS sequence $r_{l, n_{S}}(m)$ are mapped to complex-valued modulation symbols $a_{k, l}^{(p)}$ used as reference symbols for antenna port $p$ in slot $n_{s}$, where $r_{l, n_{s}}(m)$, and $a_{k, l}^{(p)}$ the mapping function between them are defined in Section 6.10.1 in [5]. As shown in Fig. 3 and 4, the white grid represents REs for data transmission, and the colored grid means C-RSs. The shaded grid denotes unused REs, because REs used for C-RS transmission on any of the other antenna ports shall not be used for transmission and are set to zero. 


\section{Channel Estimation Algorithms}

Different channel estimation theories have been used to estimate the channel response in LTE DL, such as LS, MMSE and maximum likelihood (ML), based on the availability of channel statistical information. In the proposed C-RS-aided scheme, channel response on C-RS is estimated using LS estimation and two major noise reduction techniques are used to mitigate the added noise effect at the receiver side: EWA and Wiener filtering. Instead of using 2-D Wiener filtering to do both noise reduction and interpolation, we propose using 2 1-D Wiener filtering and linear interpolation to estimate the channel response on data REs using the estimated channel response of C-RSs.
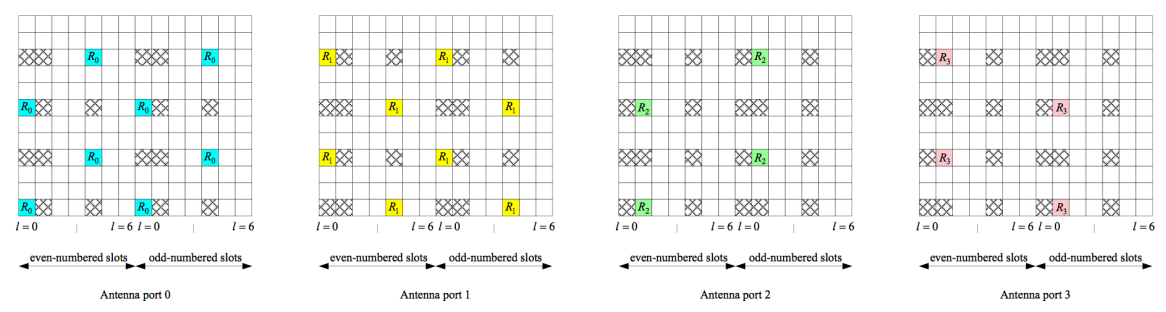

Fig. 3. Mapping of C-RS in DL LTE - Normal CP [5]
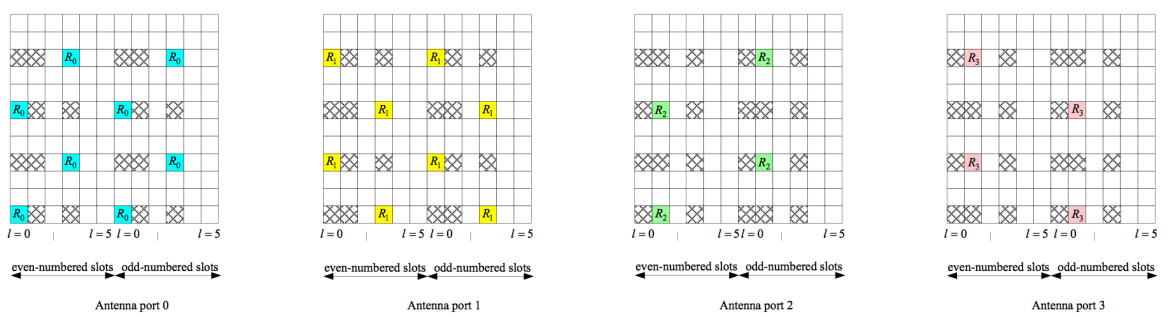

Fig. 4. Mapping of C-RS in DL LTE - Extended CP [5]

First, C-RS symbols need to be extracted from the RG. By thoroughly analyzing the pilot symbols distribution scheme proposed by [5], we arrived at mathematical equations that describe C-RS pilots locations for each antenna port, which is shown in Table 1. The locations of pilots in the RG can be written as

\section{$R G[$ Subcarrier Index, OFDM Symbol Index $]$}

Table 1. Mathematical equations for C-RS symbols extraction from RG

\begin{tabular}{|c|c|}
\hline$p=0$ & $R G\left[6 i+\left(3 t+v_{\text {shift }}\right) \bmod 6,\left(3+N_{C P}\right) t+\left(6+N_{C P}\right)\left[n_{s} \bmod 2\right]\right]$ \\
\hline$p=1$ & $R G\left[6 i+\left(3[(t+1) \bmod 2]+v_{\text {shift }}\right) \bmod 6,\left(3+N_{C P}\right) t+\left(6+N_{C P}\right)\left[n_{s} \bmod 2\right]\right]$ \\
\hline$p=2$ & $R G\left[6 i+\left(3\left[n_{s} \bmod 2\right]+v_{\text {shift }}\right) \bmod 6,1+\left[N_{C P}\left[n_{s} \bmod 2\right]\right]+6\left[n_{s} \bmod 2\right]\right]$ \\
\hline$p=3$ & $R G\left[6 i+\left(3+3\left[n_{s} \bmod 2\right]+v_{\text {shift }}\right) \bmod 6,1+\left[N_{C P}\left[n_{s} \bmod 2\right]\right]+6\left[n_{s} \bmod 2\right]\right]$ \\
\hline$v_{\text {shift }}=N_{I D}^{\text {Cell }} \bmod 6, \quad N_{C P}=\left\{\begin{array}{ll}1, & \text { For Normal Cyclic Prefix }(C P) \\
0, & \text { For Extended Cyclic Prefix }(C P)\end{array}\right\}$ \\
$i=0, \ldots, 2 \times N_{R B}^{\max , D L}-1$, & $t=0,1, \quad n_{s}=\{1,2\}$ \\
\hline
\end{tabular}


$N_{I D}^{\text {Cell }}$ is a number that identifies the cell in the network, $v_{\text {shift }}$ is the cell-specific frequency shift, $N_{R B}^{\max , D L}$ is the maximum number of DL RBs, and $n_{s}$ is the time slot number. To verify the distributions shown in Fig. 3 and 4 , set $N_{I D}^{\text {Cell }}=0$. So, after CP removal and applying the FFT, the received frequency domain C-RS signal can be represented as

$$
Y_{l, k}=H_{l, k} X_{l, k}+W_{l, k}
$$

where $Y_{l, k}$ represents received symbol, $H_{l, k}$ represents ideal channel response, $W_{l, k}$ denotes Additive White Gaussian Noise (AWGN) with zero mean and variance $\sigma_{\omega}^{2}$, and $X_{l, k}$ denotes a transmitted frequency domain C-RS signal at the $k^{\text {th }}$ subcarrier of the $l^{\text {th }}$ symbol.

Now, LS equalization is used to estimate the pilots and noise reduction techniques are applied to enhance the estimation. Noise reduction is needed since LS estimation requires no knowledge about the channel statistics, and thus, the estimation performance is generally not good enough. C-RS locations and values are known to both receiver and transmitter. Using LS estimation to solve for $H_{l, k}$ from (1) at locations where pilots were originally sent; $P_{l, k}$, we get

$$
\widehat{H}_{L S ; l, k}=\frac{Y_{l, k}}{P_{l, k}}=H_{l, k}+\widetilde{W}_{l, k}
$$

where $P_{l, k}$ represents known transmitted C-RS symbol, $\widehat{H}_{L S ; l, k}$ represents LS estimated actual channel response for locations occupied by pilot symbols and $\widetilde{W}_{l, k}$ represents the associated AWGN noise.

\subsection{Equal Weight Averaging (EWA)}

Without statistical knowledge, LS estimates can be treated as deterministic but unknown. In this case, LS estimation is followed by equal weight averaging according to following

$$
\begin{gathered}
\widehat{H}_{A V G ; n, k}=\frac{1}{Z} \sum_{n-\frac{F}{2}}^{n+\frac{F}{2} k+\frac{T}{2}} \widehat{H}_{L S ; n, k} \\
Z=\text { length }\left(\left.\widehat{H}_{L S ; n, k}\right|_{k-\frac{F}{2} \& n-\frac{F}{2} \& n+\frac{T}{2}} ^{k} \neq 0\right)
\end{gathered}
$$

where $F$ is the frequency window size and $T$ is the time window size. Fig. 5 shows an example of different EWA schemes. Note that EWA is applied for each antenna port separately; meaning that the averaging window spans pilots from one antenna port at a time. 


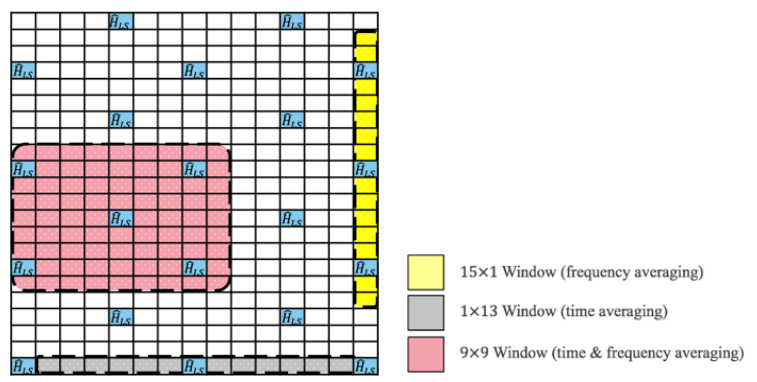

Fig. 5. Example of EWA

\subsection{Wiener Filtering}

If channel statistics are available, the estimation performance can be greatly improved by exploiting this statistical information. Thus, the LS estimates are considered as random variables and a linear filter based on the MMSE theory is used for noise reduction, namely Wiener filter. We are trying to find the channel estimates $\widehat{H}_{W ; n, k}$ from the noisy channel measurements $\widehat{H}_{L S ; n, k}$ with properly chosen estimator coefficients $b_{v, m}$ using a linear estimator that can be written as

$$
\widehat{H}_{W ; n, k}=\sum_{i, m} b_{i, m} \widehat{H}_{L S ; n, k}
$$

To simplify the formalism, we assume that only a finite number of $V$ samples $\widehat{H}_{W ; n, k}$ must be estimated from a finite number $M$ of measurements $\widehat{H}_{L S ; n, k}$. We may then write the linear estimator as

$$
\begin{gathered}
\widehat{\boldsymbol{H}}_{W}=\boldsymbol{B} \widehat{\boldsymbol{H}}_{L S} \\
\boldsymbol{B}=\left(\begin{array}{ccc}
b_{11} & \cdots & b_{1 M} \\
\vdots & \ddots & \vdots \\
b_{V 1} & \cdots & b_{V M}
\end{array}\right)
\end{gathered}
$$

The criteria for designing the Wiener filter coefficients $\boldsymbol{B}$ is

$$
E\left\{\left|e_{n, k}\right|^{2}\right\}=E\left\{\left|H_{n, k}-\widehat{H}_{W ; n, k}\right|^{2}\right\}
$$

Based on the MMSE theory, we arrive at the Wiener-Hopf equation that describes the values of $\boldsymbol{B}$ as

$$
\boldsymbol{B}=\boldsymbol{R}_{\hat{\boldsymbol{H}}_{W} \hat{\boldsymbol{H}}_{L S}} \boldsymbol{R}_{\boldsymbol{H}_{L S} \boldsymbol{H}_{L S}}^{-1}
$$

where $\boldsymbol{R}_{\boldsymbol{H}_{W} \boldsymbol{H}_{L S}}$ is the cross-correlation matrix between $\widehat{\boldsymbol{H}}_{W}$ and $\widehat{\boldsymbol{H}}_{L S}$, and $\boldsymbol{R}_{\boldsymbol{H}_{L S} \boldsymbol{\boldsymbol { H }}_{L S}}$ is the inverse of the channel auto-correlation matrix. We assume that the noise and the fading are statistically independent. Assuming the WSSUS model for $H_{n, k}$ with twodimensional autocorrelation function; $\mathcal{R}(f, t)$, and that the noise and the fading are statistically independent, then the matrix elements of $\boldsymbol{R}_{\boldsymbol{H}_{L S} \boldsymbol{H}_{L S}}$ and $\boldsymbol{R}_{\boldsymbol{H}_{W} \boldsymbol{H}_{L S}}$ are given by, respectively 


$$
\begin{gathered}
\left(\boldsymbol{R}_{\boldsymbol{H}_{L S} \boldsymbol{H}_{L S}}\right)_{q m}=\mathcal{R}\left(f_{q}-f_{m}, t_{q}-t_{m}\right)+\delta_{q m} \gamma \\
\left(\boldsymbol{R}_{\hat{\boldsymbol{H}}_{W} \boldsymbol{H}_{L S}}\right)_{v m}=\mathcal{R}\left(f_{v}-f_{m}, t_{v}-t_{m}\right)
\end{gathered}
$$

and $\gamma=\sigma_{x}^{2} / \sigma_{\omega}^{2}$ is the SNR. For wide-sense stationary uncorrelated scattering (WSSUS) channels [10], the scattering function and thus the two-dimensional autocorrelation function factorizes to

$$
\mathcal{R}(f, t)=\mathcal{R}(f, 0) \mathcal{R}(0, t)=\mathcal{R}_{H}(f) \mathcal{R}_{c}(t)
$$

$\mathcal{R}_{c}(t)$ is given as the inverse Fourier transform of the Doppler spectrum. For the Jakes spectrum with $D_{\max }$ as the maximum Doppler frequency, it is given by

$$
\mathcal{R}_{c}(t)=J_{0}\left(2 \pi D_{\max } t\right)
$$

$\mathcal{R}_{H}(f)$ is given as the Fourier transform of the delay power spectrum. For a rectangular delay power spectrum between 0 and $\tau_{\max }$, it is given by

$$
\mathcal{R}_{H}(f)=e^{-j \pi f \tau_{\max }} \cdot \operatorname{sinc}\left(f \tau_{\text {max }}\right)
$$

Different variations of Wiener filtering can be used such as: 1-D Frequency Wiener, 1-D Time Wiener, 2 1-D Wiener and 2-D Wiener. We have tested 2-D Wiener filtering with linear interpolation before [9]. In the same manner, mathematical equations can be written for the other types of Wiener filtering. 2-D Wiener filtering provides the best noise reduction. However, instead of that rather involved 2-D filtering, one can perform a suboptimal 1-D $\times 1$-D (2 1-D) filtering without losing significantly in performance. Thus, 2 1-D Wiener filtering has been suggested as an alternative to 2-D Wiener filtering [10]. It is done by applies the two different modes of 1D Wiener filters back to back as shown in Fig. 6 . The order can be shown to be arbitrary due to linearity of the estimation and due to the fact that the rectangular constellation is the Cartesian product of two one-dimensional ones [10].

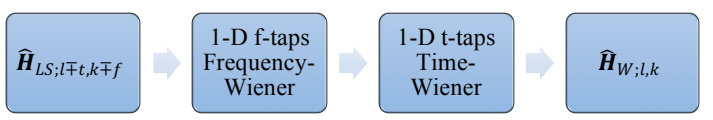

Fig. 6. 2 1-D Wiener filtering procedure

\section{Simulation Results and Analysis}

Simulations were carried out to compare different types of EWA (different window sizes) with Wiener filtering in different channel conditions. We considered three delay profiles: Extended Typical Urban model (ETU), Extended Vehicular A model (EVA) and Extended Pedestrian A model (EPA) [5], and they represent a low, medium, and high delay spread environment, respectively. The specifications for the multipath delay profiles for the simulated channels are shown in Table 2. Comparisons in terms of performance and complexity are carried out between the proposed scheme, 2 1-D Wiener filtering and linear interpolation, and three other major schemes: 2-D Wiener 
filtering and Wiener interpolation, 2-D Wiener filtering and linear interpolation, and EWA (with different window sizes) and linear interpolation.

Table 2. Channel delay profiles used in simulation

\begin{tabular}{|c|c|c|}
\hline Delay Profile & Doppler Spread $(\mathrm{Hz})$ & Max. Excess Tap Delay $(\mathrm{ns})$ \\
\hline EPA & 5 & 410 \\
\hline EVA & 70 & 2510 \\
\hline ETU & 300 & 5000 \\
\hline
\end{tabular}

The complexity analysis for C-RS based pilots is carried for a frequency time plane spanning 1200 subcarriers and 14 OFDM symbols. Fig. 8, 9 and 10 show the MSE between the interpolated CFR values and the actual CFRs for ETU300, EPA5 and EVA70, respectively, using different noise reduction schemes. It is noted that Wiener filtering and Wiener interpolation outperforms all other schemes for the different channel modes, but that comes at the cost of higher number of computations as shown in Table 3 for C-RS $p=0$. Each complex multiplication operation translates to four real multiplication operations. The proposed scheme, 2 1-D Wiener filtering with linear interpolation, has relatively lower computational complexity when compared to 2-D Wiener filtering with linear interpolation, and thus will focus the discussion comparing it to the different versions of equal weight averaging, which is of relatively similar computational complexity.

Table 3. Comparison of noise reduction techniques complexity for C-RS $p=0$

\begin{tabular}{|c|c|c|c|c|}
\hline Algorithm & $\begin{array}{c}\text { EWA + Linear } \\
\text { Interp. }\end{array}$ & $\begin{array}{c}\text { 2-DWiener Filtering } \\
\text { and Interp. }\end{array}$ & $\begin{array}{c}\text { 2 1-D Wiener Filtering + } \\
\text { Linear Interp. }\end{array}$ & $\begin{array}{c}\text { 2-D Wiener Filtering + } \\
\text { Linear Interp. }\end{array}$ \\
\hline Est. \# of & $200 * 32 * 4+$ & $1200 * 32 * 14=$ & $200 * 10 * 4=8000 \mathrm{CO}$ & $200 * 32 * 4=25,600 \mathrm{CO}$ \\
Operations & $1200 * 14=$ & $537,600 \mathrm{CO}=$ & $=32,000 \mathrm{RO} \& 1200 *$ & $=102,400 \mathrm{RO} \& 1200 *$ \\
Real Op (RO) & $30,400 \mathrm{RO}$ & $2,150,400 \mathrm{RO}$ & $14=16,800 \mathrm{RO}$ & $14=16,800 \mathrm{RO}$ \\
Complex OP (CO) & & \multicolumn{1}{|c|}{ RO } \\
\hline
\end{tabular}

Fig. 8 shows the simulation results for ETU300 channel delay profile, and the results suggest that the best low complexity scheme would be a combination of both EWA and Wiener filtering with linear interpolation. Moreover, it can be seen that Wiener filtering adapts well to channel conditions variations compared to EWA. Although EWA is computationally less costly, averaging window size must be chosen very carefully based on the value of SNR. Compare to 2-D Wiener with linear interpolation, 2 1-D Wiener with linear interpolation is $\sim 5 \mathrm{~dB}$ worse in terms of SNR as shown in Fig. 8.

Fig. 9 shows the simulation results for EVA70 channel delay profile. The $53 \times 27$ EWA performs better than the proposed scheme for very low SNR, but after SNR $-3 \mathrm{~dB}$, the proposed scheme performs better noise reduction. However, compared to 2-D Wiener, we are sacrificing $\sim 3 \mathrm{~dB}$ in SNR in terms of performance.

Fig. 10 shows the simulation results for EPA5 channel delay profile. As SNR increases, averaging schemes with bigger window size introduces a smearing effect, which gives worse results compared to the LS estimates. Moreover, after SNR $\cong 12$ $\mathrm{dB}, 2$-D Wiener filtering with linear interpolation becomes worse than the $13 \times 27$ equal weight averaging, due to the high number of taps used for noise reduction, which smears the signal at high SNR. 2 1-D Wiener performs better than EWA up till SNR 
$\cong 10 \mathrm{~dB}$, where EWA with $13 \times 27$ outperforms 2 1-D Wiener. So, b y using 2 1-D Wiener instead of 2-D, we sacrifice $\sim 2 \mathrm{~dB}$ in SNR.

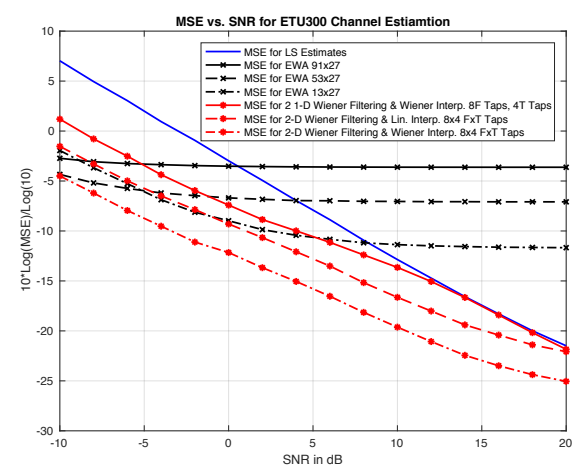

Fig. 8. Channel Estimation for ETU300

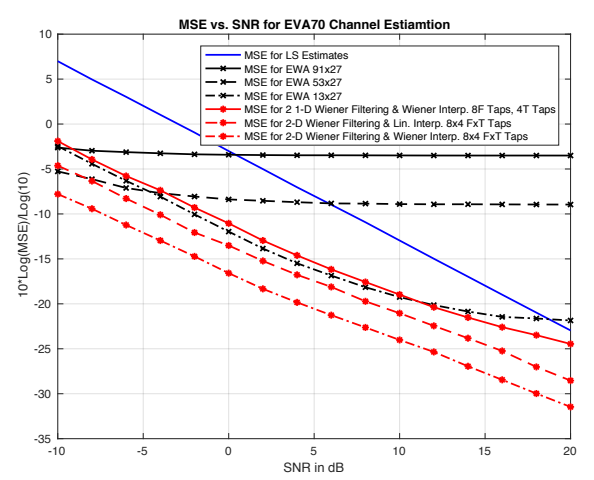

Fig. 9. Channel Estimation for EVA70

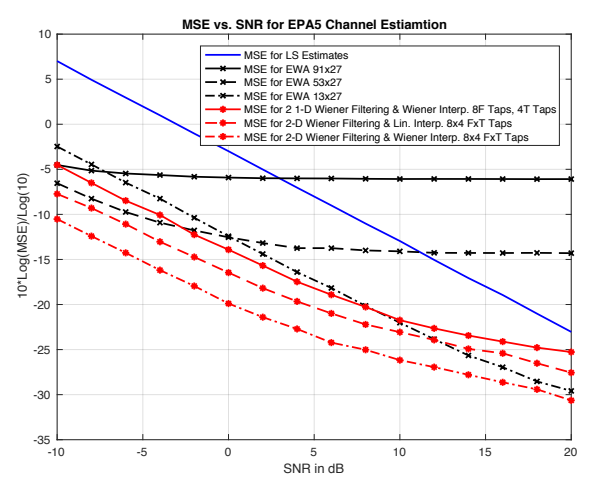

Fig. 10. Channel Estimation for EPA5

The degradation in performance when using 2 1-D Wiener instead of 2-D Wiener for noise reduction for C-RS symbols varies as channel variations increase. One more disadvantage of Wiener filtering compared to EWA is the fact that the memory usage 
is considerably higher when using Wiener, as we need to store the $\boldsymbol{B}$ coeffiecents for different combinations of delay profiles and SNR values in order to avoid calculating them on the fly, which introduces great computational complexity because of the inversion process associated with the auto-correlation matrices.

\section{CONCLUSIONS}

A low complexity C-RS-aided channel estimation scheme is presented for the coherent detection for LTE DL OFDM communication systems. The proposed scheme substitutes 2-D Wiener filtering with linear interpolation with 2 1-D Wiener filtering and linear interpolation. From the simulation analysis carried out, the proposed scheme trades off performance for computational complexity. 2 1-D Wiener filtering with linear interpolation requires $\sim 41 \%$ of the number of computations needed by 2 -D Wiener filtering with linear interpolation. Moreover, mathematical equations that describe CRS symbols extraction from the RG for antenna ports $0,1,2$ and 3 were presented. Simulations with different window sizes in EWA showed an inverse relationship with the SNR value. The best noise reduction method is a combination of both averaging and Wiener filtering with linear interpolation, where the lower bound is a function of both SNR and the channel statistics.

\section{References}

1. Hou, J., Liu, J.: A novel channel estimation algorithm for 3GPP LTE downlink system using joint time-frequency two-dimensional iterative Wiener filter. In: IEEE International Conference on Communication Technology (November 2010)

2. 3GPP TR 36.814 V0.4.1, Further Advancements for E-UTRA Physical Layer Aspects (Release 9) (February 2009)

3. Bahai, A.R.S., Saltzberg, B.R.: Multi-Carrier Digital Communication: Theory and Applications of OFDM. Kluwer Academic/Plenum (1999)

4. Van de Beek, J.-J., Edfors, O., Sandell, M., Wilson, S.K., Borjesson, P.O.: On channel estimation in OFDM systems. In: IEEE 45th Vehicular Technology Conference, pp. 815-819 (1995)

5. 3GPP TS 36.211 V9.1.0, Technical Specification Group Radio Access Network for E-UTRA Physical channels and Modulation (Release 9) (March 2010)

6. Qin, Y., Hui, B., Chang, K.H.: Performance evaluation of pilot-based channel estimation and equalization techniques in 3GPP LTE downlink. In: Proc. Of KICS Summer Conference (November 2009)

7. Zhou, W., Lam, W.H., A novel method of Doppler shift estimation for OFDM systems. In: IEEE Military Communication Conference (November 2008)

8. Recommendation ITU-R M.1225, Guidelines for Evaluation of Radio Transmission Technologies for IMT-2000(Question ITU-R 39/8) (1997)

9. Zourob, M., Rao, R., Reduced-Complexity Implementation Scheme for OFDM Channel Estimation. Submitted in CCECE 2017

10.Schulze, H., Luders, C.,: Theory and Applications of OFDM and CDMA. Wiley. West Sussex, England (2005) 